\title{
Child and Adolescent Social Work Journal Editorial Policy: Guidelines for Authors
}

\author{
Lisa Schelbe ${ }^{1} \cdot$ Bruce A. Thyer $^{1}$ \\ Published online: 12 December 2018 \\ (c) Springer Science+Business Media, LLC, part of Springer Nature 2018
}

What is the process to publish in the Child and Adolescent Social Work Journal (CASWJ)? How should I structure my manuscript for the CASWJ? What advice do you have for someone preparing a manuscript for the CASWJ? These are some of the questions that you may have as you determine if you wish to prepare a manuscript for publication in the CASWJ. This editorial provides guidance, demystifies the process, and answers questions about publishing in CASWJ.

Let us begin with a brief overview of the CASWJ. Over 3 decades old, the CASWJ features original articles that focus on social work practice with children, adolescents, and their families. The journal addresses current issues in the field of social work drawn from theory, direct practice, research, and social policy. The range of topics includes problems affecting a variety of specific populations in special settings. CASWJ welcomes a range of scholarly contributions focused on children and youth, including theoretical papers, narrative case studies, historical analyses, traditional reviews of the literature, descriptive studies, single-system research designs, correlational investigations, methodological works, pre-experimental, quasi-experimental and experimental evaluations, meta-analyses and systematic reviews. Appropriate fields of practice include interpersonal practice, small groups, families, organizations, communities, policy practice, nationally-oriented work, and international studies. Manuscripts involving qualitative, quantitative and mixed methods are welcome to be submitted, as are papers grounded in one or more theoretical orientations, or those that are not based on any formal theory. To get a sense of CASWJ publications, you may wish to review recent issues. Inquiries regarding the potential suitability of your paper may be directed to either Co-Editor. The journal does not have any page limits, but requires that all submissions be formatted in strict compliance with all aspect of the current

Lisa Schelbe

lschelbe@yahoo.com

1 Florida State University, Tallahassee, USA
APA style. Manuscripts should be submitted online at http:// www.editorialmanager.com/casw.

\section{Before Writing a Manuscript}

At the point in which you are reading this editorial, you may have a completed study and manuscript and are looking for an outlet for your work, or you may still be in the beginning stages of research. If this is the case, CASWJ would like to encourage you to consider adopting practices that are increasingly being recognized in contributing to transparency in research.

You may wish to preregister your research plans (also known as a protocol). Gonzales and Cunningham (2015) describe the benefits of preregistration including the decrease of a publication bias. The Open Science Framework (OSF; Center for Open Science, 2018) is one resource for registration, although there are many others, especially for clinical trials such as ClinicalTrials.gov (U.S. National Library of Medicine, 2018) and those identified by the World Health Organization (2018) to be part of the International Clinical Trials Registry Platform. Usually used for intervention studies, clinical trial registries are where you describe the purposes of your study, the research question(s), hypotheses, outcome measures, intervention(s), and plans for data analysis. Once placed in the public domain, when you eventually publish your completed project readers can compare what you actually did, relative to what you previously said you were going to do. This transparency helps keeps researchers honest. For example, if you preregistered a protocol and said you were going to have five outcome measures, but in your final report you only included results from two outcome measures, alert readers would wonder what happened to the missing measures. Unscrupulous researchers might have used the original five outcome measures but found that only two supported their hypotheses and decided to publish only the positive results. This is, in effect, cherry 
picking the data, only reporting favorable results, when good science would require that you report all the results, positive and negative. You are less likely do to this, and get away with it, if your protocol is preregistered. Another sin committed by some is to have one study with several outcome measures, and instead of preparing one paper with the results from all measures, prepare individual papers with each reporting only on one of the several outcome measures. This unethical way of inflating one's curriculum vitae gives rise to the appearance that more studies in a given area have been conducted that is actually the case. It may require a keen-eyed reader to track down all the separate papers, published in different journals, from this one study. If you are preparing a systematic review, you are encouraged to prospectively register your systematic review to be in compliance with PRISMA standards and as a safeguard against reporting bias (Stewart, Moher, \& Shekelle, 2012).

Once published you are encouraged to use data-depositories so that your data is available after publication. This can be as simply as downloading your SPSS database and coding manual to some public site. This permits other scholars to access your data, check the accuracy of your analyses, and perhaps extend them. Again, the transparent nature of scientific inquiry helps keep us all honest and by preregistering our study protocol prior to beginning data collection, and then making all our data publicly available, researcher bias can be reduced and errors uncovered more readily. For a review of the benefits and risks of making data available as well as an example, see Wicherts and Bakker (2012). Fecher, Friesike, and Hebing (2015) provide information about the process of data sharing. See Carroll (2015) for a review of legal considerations of making data publicly available.

\section{Journal Article Reporting Standards}

In the interest of promoting transparent rigorous research, CASWJ endorses the use of recognized journal article reporting standards. Specifically, CASWJ requires that manuscripts follow the Journal Article Reporting Standards (JARS) developed by the APA Publications and Communications Board Task Force (Appelbaum et al., 2018; Levitt et al., 2018). The original JARS first appeared in the 6th edition of the APA manual as an appendix (APA, 2009, pp. 247-253), and provided a checklist of information that should be included in all empirical research reports, and additional standards for studies that introduced an intervention, those that made use of quasi-experimental, experimental and meta-analysis methodologies. An example of a flow chart of participants in an experiment or quasi-experiment was also provided and recommended to include as a figure in such studies.

\section{Guidelines for Quantitative Studies}

The JARS Quantitative Standards (JARS-Quant) recently were updated and include specific guidelines for different types of quantitative studies (Appelbaum et al., 2018). A simple flowchart is included so you can determine which guidelines are appropriate for your particular quantitative study. This is a more comprehensive listing that that found in the original JARS and includes standards for reporting on single-subject research studies, a new addition to the JARS. Once you determine which table to use, follow the guidelines presented about the information recommended to include in the manuscript.

As part of the JARS-Quant guidelines (Appelbaum et al., 2018), randomized control trials (RCTs) of interventions must include a completed checklist following the Consolidated Standards of Reporting Trials (CONSORT) checklist. Please note that CONSORT-SPI 2018 and other CONSORT guidelines are tools that will continue to evolve with new evidence and changes in scientific consensus (Montgomery et al., 2018). The newly released CONSORT-SPI 2018 (co-authored by social workers with expertise in intervention research) is specifically designed for social and psychological intervention RCTs and is an official extension of the CONSORT 2010 Statement. The CONSORT-SPI 2018 checklist extends over a third of the items in the CONSORT 2010 to provide improved reporting necessary to evaluate studies of psychosocial interventions (Montgomery et al., 2018). If you conduct a RCT, please include a checklist as described in the CONSORTSPI 2018. You may also wish to consult the CONSORT 2010 (also developed with social worker input) guidelines for studies in biomedical interventions as appropriate (Moher et al., 2012; Schulz, Altman, \& Moher, 2010).

One aspect of the JARS-Quant we would like to highlight is the recommendation that inferential statistical analyses not only report exact $p$ values, but that these $p$ values should be accompanied by their associated effect sizes and 95\% confidence intervals surrounding these effect sizes. This additional bit of statistical reportage helps ensure that statistically reliable but clinically trivial differences, associations or changes are not given undue weight, Given a sufficiently large sample size many small changes or differences will be statistically 'significant' but of absolutely no practical value. At the CASWJ we regularly request that authors provide this information when we invite a revision, and the failure to do so will likely result in the revision being returned so that this information can be included. It is increasingly being recognized that significant $p$ values alone do not make an important finding. 


\section{Guidelines for Qualitative and Mixed Methods Studies}

The JARS Qualitative Standards (JARS-Qual) are a new addition to the JARS guidelines (Levitt et al., 2018). The guidelines outline the relevant paper sections and describe the information that should be included. The JARS Qualitative Standards also outline requirements for qualitative meta-analyses. Mixed methods studies should follow the relevant JARS Standards outlined in the JARS-Qual and JARS-Quant guidelines. The use of widely adopted standardized checklists to help ensure that information crucial for a reader's understanding of your paper will go far to enhancing the transparency and reproducibility of social work research.

\section{APA Formatting}

Manuscripts must be written in English and in current APA format (APA, 2009). If your native language is not English, we recommend you have your manuscript proofread by English language experts prior to submission. There are commercial editing services which international scholars can make use of in this regard. Likewise, if you are unfamiliar with APA formatting, consider consulting with someone who can edit your manuscript to ensure it is APA compliant. There are free online guides to APA formatting available at https://www.apastyle.org/ (APA, 2018).

Be sure that you adhere to the conventions outlined in the APA publication manual in preparing your manuscript for submission to a journal. DO NOT use articles published in the CASWJ or any other journal as a guide as to how you should format your paper. When manuscripts are typeset for publishing as an actual article there can be many differences in how the paper looks. For example, many journals publish their pages using a two-column format, with page numbers at the bottom of the page and centered. In APA style we do not use two or more columns and page numbers go in the upper right hand corner of each page. In published articles, tables and figures are positioned in the appropriate place in the text. In preparing an APA journal manuscript, tables go, one per page, after the references. Figures if any, also go one per page, after the tables. Sometimes journal articles are typeset using single spacing. In preparing your manuscript you should only use double spacing. Some journals make their article titles in bigger type and in boldface. In APA style for manuscripts everything is the same typeface and font size (Times New Roman, 12 cpi is common). In other words, do not try and make your manuscript look like a published journal article. Follow the APA manual only. We do not recommend any other resource, because sometimes they get it wrong.

\section{Potential Problems}

Before we transition to talk about the processes related to publishing a manuscript with CASWJ, we want to share a few potential problems we see with manuscripts that contribute to their receiving negative reviews and potentially being rejected. As previously mentioned, CASWJ focuses on social work practice with children, adolescents, and their families. The journal publishes manuscripts on a broad range of current issues in the field of social work drawn from theory, direct practice, research, and social policy. Manuscripts must address children, adolescents, and their families directly. Those which do not are not a good fit for CASWJ and should not be submitted to CASWJ. Often you can determine if your manuscript is a good fit through looking at previous issues of the journal. If you are having a hard time determining if it is a good fit, you can also reach out to one of the co-editors.

Even manuscripts that fit within the scope of CASWJ sometimes are problematic. Reviewers often raise concerns about a disconnection between the discussion section and the introduction and literature used to frame the study. The discussion section should not simply be a reiteration of the results. Rather you should use the discussion to further the readers' understanding on the topic. The discussion should situate your findings within the broader literature on the topic. Please note that following the JARS guidelines will assist in ensuring the appropriateness of your discussion.

Reviewers frequently comment upon the implications of the study. Specifically, a concern often raised is the lack of an implications section, implications unrelated to the results, and overstated implications. Implications unrelated to results often are in the form of specific recommendations to use interventions that were not addressed in the study. For example, a study with the key finding about a specific population of parents lack of social support contributing to children's poor outcomes may suggest the need for interventions that provide social scaffolding and support. However, to identify specific programs that have not been empirically tested with the group is questionable. Make sure that your implications are supported by the findings, yet are not overstated. For example, a smallscale exploratory study about foster care should have humble recommendations and suggestions for future research rather than stating that the findings support a radical overhaul of child welfare system.

If your study has strong conclusions you may wish to go beyond mentioning implications to suggesting potential applications. For example, if your outcome study is the latest in a long line to investigations which found that a given psychosocial intervention or assessment measure 
does not work, you may wish to state that one potential application of your findings is that the assessment method or intervention you investigated should stop being used by social workers. This is rarely done in social work, but there are some examples. Our literature and those of related fields have been unembarrassed to suggest that therapies intended to change a person's sexual orientation do not work and should not be used. Other examples include the bogus treatment called facilitated communication, wherein a person with autistic spectrum disorder has their hand held over a keyboard while they are said to type. The controlled literature is crystal clear in showing that that it has been the facilitator unknowingly guiding the client's hand, and not the client originating the communicating. Restrictive holding therapies coercively applied to noncompliant children are another example of so-called therapies which evidence suggests should not be used. See Thyer and Pignotti (2015) and Holden and Barker (2018) for numerous examples of treatments which research suggests should not be used. More positive research evidence can be used to suggest potentially useful therapies which should be used, when this information is incorporated with clients' preferences and values, professional ethics, one's own clinical expertise, available resources, costs, and the other elements which go into the decision-making process known as evidence-based practice.

\section{Submission Process}

After you have written your manuscript following proper structure and proofread it carefully, the next step is to prepare your manuscript for submission. CASWJ follows a double-blind review process, so your manuscript should not include any of its authors' and affiliation in the text of the document. The CASWJ website "Instructions for Authors" outlines the details for submission, including required statements about compliance with ethical standards, and providing information about potential conflicts of interest. In preparing your online submission, please use the website's information as the current authority, as publisher policies may change after this editorial is published.

When you submit your manuscript through the online submission portal, your manuscript will be assigned a manuscript identification number. Anytime you communicate with the co-editors or the journal staff about the manuscript, make sure to include the number.

\section{Review Process}

Manuscripts are sent out to reviewers who are asked to provide detailed feedback. At the CASWJ we ask our Editorial Board members to self-select which papers they review. We rarely assign reviewers. This helps assure that your paper is reviewed by someone with a genuine interest in the topic of your research. The reviewers do not know your identity, gender, affiliation or anything else about you. Reviewers provide the Co-Editors with suggestions to accept your paper, to revise it with minor changes, to revise it with major changes, or to decline to publish it. If one of the reviewers suggests that your paper be declined, we almost always follow that suggestion. In this manner we try and assure that only research of unambiguously high quality gets accepted and published. It is not a perfect system. Sometimes we turn away papers that perhaps should have been published, and publish those unworthy of gracing the pages of the journal. It is frustrating when we make such mistakes, but they are always made using our best judgement and we think these errors are pretty rare.

In some cases, when the reviewers do not recommend publication in the CASWJ, we send your manuscript to the Springer Transfer Desk. This is typically done with manuscripts that have scientific merit, but are not appropriate for publishing in the CASWJ for some reason. Staff at the transfer desk assist authors in finding another suitable Springer journal (2018). This service is free and voluntary.

\section{Revise and Resubmit Process}

If you receive the decision "Revise and Resubmit," you will typically be given 30 days to submit a revision. When you submit your revision, you will also be required to submit a separate cover letter describing how you addressed each substantive suggestion made by the reviewers. Please note how you addressed the specific feedback in your letter as Reviewer 1, point \#1, point \#2, etc., Reviewer 2, point \#1, etc. In the letter it is helpful if you include page numbers where changes were made and examples of the changes made as appropriate.

In your revised manuscript, you need not necessarily comply with all the suggestions made by reviewers. However, provide some rationale for your decision if you elect not to. It is not necessary to use track changes or highlight the changes made in the revised manuscript. Please submit your revision as a 'clean' manuscript and put in any previously removed citations taken out for the purposes of 'blinding' the original submission.

\section{After Acceptance}

When your manuscript is accepted, you will receive an email congratulating you and details that it is transferred to production. It is a courteous practice for you to notify your co-authors, if any. An email will be sent asking for you 
to complete the copyright information. A timely response to these emails and the process is important. After signing forms electronically on behalf of your co-authors and you, the manuscript will be sent to the copy editors. You will receive a link to review the manuscript and address comments and questions of the copy editors. Please do not ask for any changes to be made in the author and publisher's copyright agreement. With hundreds of journals being produced, publishers cannot constantly amend their standard agreements to satisfy the quirks and idiosyncrasies of their myriad authors.

During the process of publishing you will have the opportunity to make your article open access, meaning it will be available to all, including people who do not have subscriptions to CASWJ as individuals or through an institution. Open access publishing is required by some grant funders and foundations. There is evidence that open access articles are cited more than those which are not (e.g., Piwowar et al., 2018). There is a fee that applies to have your article be open access. Currently, it is $\$ 3000$. The decision to have your article open access is yours. Most of our authors lack the wherewithal to pay the open access fee.

Once your proofs are complete and finalized by Springer staff, your article will be available in Online First, the journal's website this version has a Digital Object Identifier (doi) and will be searchable through various search engines. When your article is assigned to a specific issue of CASWJ, you will receive an email notifying you. You will also receive an author's copy of the print issue your article appeared in.

When your article is published you will also receive a SharedIt link (Springer Nature, 2018). This is a service that SpringerNature provides that offers a view-only version of your article. Anyone who has this link can read your article online. You are encouraged to post this link to help disseminate your research. It can be shared on social media sites, websites, and scholarly collaborative networks.

Another resource to disseminate your publication is using institutional repositories. Institutional repositories are recognized as a strategy to promote scholarly communication and can assist with the dissemination of research (Lynch, 2003). They are beneficial in that institutional repositories can increase visibility of work (Swan, 2016). Check with your university or college library to learn what options are available to you. Make sure to follow publisher guidelines when using institutional repositories.

\section{Conclusion}

CASWJ is committed to quality articles focusing on social work practice with children, adolescents, and their families. If you are considering CASWJ as a journal for your manuscripts, review recent articles in the journal and become familiar with the requirements and publication process. Before submitting your manuscript, make sure to proofread it including checking that it strictly followed APA guidelines. Remember to use the appropriate JARS guidelines. We are committed to working with reviewers to provide timely and meaningful feedback to strengthen the manuscript. We look forward to your submission.

\section{References}

American Psychological Association (APA). (2009). Publication manual of the American Psychological Association (6th ed.). Washington, DC: American Psychological Association.

American Psychological Association (APA). (2018). APA Style Central. Retrieved Oct 1, 2018, from https://www.apastyle.org/.

Appelbaum, M., Cooper, H., Kline, R. B., Mayo-Wilson, E., Nezu, A. M., \& Rao, S. M. (2018). Journal article reporting standards for quantitative research in psychology: The APA Publications and Communications Board task force report. American Psychologist, 73, 3-25.

Carroll, M. W. (2015). Sharing research data and intellectual property law: A primer. PLoS Biology, 13(8), e1002235. https://doi. org/10.1371/journal.pbio.1002235.

Center for Open Science. (2018). Open science framework. https ://osf.io/.

Fecher, B., Friesike, S., \& Hebing, M. (2015). What drives academic data sharing? PLoS ONE, 10(2), e0118053. https://doi. org/10.1371/journal.pone.0118053.

Gonzales, J. E., \& Cunningham, C. A. (2015). The promise of pre-registration in psychological research. Psychological Science Agenda. Retrieved https://www.apa.org/science/about/ psa/2015/08/pre-registration.aspx.

Holden, G., \& Barker, K. (2018). Should social workers be engaged in these practices? Journal of Evidence-based Social Work, 15, $1-13$.

Levitt, H. M., Bamberg, M., Creswell, J. W., Frost, D. M., Josselson, R., \& Suárez-Orozco, C. (2018). Journal article reporting standards for qualitative primary, qualitative meta-analytic, and mixed methods research in psychology: The APA Publications and Communications Board task force report. American Psychologist, 73, 26-46.

Lynch, C. A. (2003). Institutional repositories: Essential infrastructure for scholarship in the digital age. Portal: Libraries and the Academy, 3, 327-336.

Moher, D., Hopewell, S., Schulz, K. F., Montori, V., Gøtzsche, P. C., Devereaux, P. J., ... Altman, D. G. (2012). CONSORT 2010 explanation and elaboration: Updated guidelines for reporting parallel group randomised trials. International Journal of Surgery, $10(1), 28-55$.

Montgomery, P., Grant, S., Mayo-Wilson, E., Macdonald, G., Michie, S., Hopewell, S., \& Moher, D. (2018). Reporting randomised trials of social and psychological interventions: The CONSORT-SPI 2018 extension. Trials, 19(1), 407.

Piwowar, H., Priem, J., Larivière, V., Alperin, J. P., Matthias, L., Norlander, B., ... Haustein, S. (2018). The State of OA: A largescale analysis of the prevalence and impact of Open Access articles. PeerJ, 6, e4375.

Schulz, K. F., Altman, D. G., \& Moher, D. (2010). CONSORT 2010 statement: Updated guidelines for reporting parallel group randomised trials. BMC Medicine, 8, 18. 
Springer. (2018). The transfer desk. https://www.springer.com/gp/autho rs-editors/journal-author/the-springer-transfer-desk. Accessed 1 Oct 2018.

Springer Nature. (2018) SharedIt. https://www.springernature.com/gp/ researchers/sharedit. Accessed 1 Oct 2018.

Stewart, L., Moher, D., \& Shekelle, P. (2012). Why prospective registration of systematic reviews makes sense. Systematic Reviews, $1,7-11$.

Swan, A. (2016). Institutional repositories-Now and next. In P. Dale, J. Beard \& M. Holland (Eds.), University libraries and digital learning environments (pp. 145-160). New York: Routledge.

Thyer, B. A., \& Pignotti, M. (2015). Science and pseudoscience in social work practice. New York: Springer.

U.S. National Library of Medicine. (2018). ClinicalTrials.gov. Retrieved Oct 1, 2018, from https://clinicaltrials.gov/.
Wicherts, J. M., \& Bakker, M. (2012). Publish (your data) or (let the data) perish! Why not publish your data too? Intelligence, 40 , 73-76.

World Health Organization. (2018). International Clinical Trials Registry Platform. Retrieved Oct 1, 2018, from http://www.who.int/ ictrp/network/primary/en/.

Publisher's Note Springer Nature remains neutral with regard to jurisdictional claims in published maps and institutional affiliations. 